\title{
QUADRATIC-LIKE DYNAMICS OF CUBIC POLYNOMIALS
}

\author{
ALEXANDER BLOKH, LEX OVERSTEEGEN, ROSS PTACEK, \\ AND VLADLEN TIMORIN
}

\begin{abstract}
A small perturbation of a quadratic polynomial $f$ with a non-repelling fixed point gives a polynomial $g$ with an attracting fixed point and a Jordan curve Julia set, on which $g$ acts like angle doubling. However, there are cubic polynomials with a non-repelling fixed point, for which no perturbation results into a polynomial with Jordan curve Julia set. Motivated by the study of the closure of the Cubic Principal Hyperbolic Domain, we describe such polynomials in terms of their quadratic-like restrictions.
\end{abstract}

\section{INTRODUCTION}

In this paper, we study topological dynamics of complex cubic polynomials. We denote the Julia set of a polynomial $f$ by $J(f)$ and the filled Julia set of $f$ by $K(f)$. Let us recall classical facts about quadratic polynomials. The Mandelbrot set $\mathcal{M}_{2}$, perhaps the most wellknown mathematical set outside of the mathematical community, can be defined as the set of all complex numbers $c$ such that the sequence

$$
c, \quad c^{2}+c, \quad\left(c^{2}+c\right)^{2}+c, \ldots
$$

is bounded. The numbers $c$ label polynomials $z^{2}+c$. Every quadratic polynomial can be reduced to this form by an affine coordinate change.

By definition, $c \in \mathcal{M}_{2}$ if the orbit of 0 under $z \mapsto z^{2}+c$ is bounded. What is so special about the point 0 ? It is the only critical point of the polynomial $z^{2}+c$ in $\mathbb{C}$. A critical point of a complex polynomial has a meaning in the realm of topological dynamics. Namely, this is a point that does not have a neighborhood, on which the map is one-to-one. Generally, the behavior of critical orbits to a large extent determines the dynamics of other orbits. For example, by a classical theorem of

Date: November 8, 2014; revised July 21, 2015; next revision November 30, 2015.

2010 Mathematics Subject Classification. Primary 37F45; Secondary 37F10, 37F20, 37F50.

Key words and phrases. Complex dynamics; Julia set; polynomial-like maps; laminations. 
Fatou and Julia, $c \in \mathcal{M}_{2}$ if and only if the filled Julia set of $z^{2}+c$

$$
K\left(z^{2}+c\right)=\left\{z \in \mathbb{C} \mid z, z^{2}+c,\left(z^{2}+c\right)^{2}+c, \cdots \not \rightarrow \infty\right\}
$$

is connected. If $c \notin \mathcal{M}_{2}$, then the set $K\left(z^{2}+c\right)$ is a Cantor set.

The Mandelbrot set has a complicated fractal shape. Yet one can see many components of the interior of $\mathcal{M}_{2}$ bounded by real analytic curves (in fact, ovals of real algebraic curves). The central part of the Mandelbrot set, the Principal Hyperbolic Domain $\mathrm{PHD}_{2}$, is bounded by a cardioid (a curve, whose shape resembles that of a heart). This cardioid is called the Main Cardioid. By definition, the Principal Hyperbolic Domain $\mathrm{PHD}_{2}$ consists of all parameter values $c$ such that the polynomial $z^{2}+c$ is hyperbolic, and the set $K\left(z^{2}+c\right)$ is a Jordan disk (a polynomial of any degree is said to be hyperbolic if the orbits of all its critical points converge to attracting cycles). Equivalently, $c \in \mathrm{PHD}_{2}$ if and only if $z^{2}+c$ has an attracting fixed point.

The closure of $\mathrm{PHD}_{2}$ consists of all parameter values $c$ such that $z^{2}+c$ has a non-repelling fixed point. As follows from the Douady-Hubbard landing theorem [DH8485, Hub93] and Pommerenke-Levin-Yoccoz inequality Hub93, Lev91, Pom86, Pet93, the Mandelbrot set itself can be thought of as the union of the main cardioid and limbs (connected components of $\mathcal{M}_{2} \backslash \overline{\mathrm{PHD}}_{2}$ ) parameterized by reduced rational fractions $p / q \in(0,1)$. This motivates our study of cubic analogs of $\mathrm{PHD}_{2}$ started in BOPT14 and continued in BOPT14a. We begin our discussion by describing some results of these two papers.

Complex numbers $c$ are in one-to-one correspondence with affine conjugacy classes of quadratic polynomials (throughout we call affine conjugacy classes of polynomials classes of polynomials). Thus, a higherdegree analog of the set $\mathcal{M}_{2}$ is the degree $d$ connectedness locus $\mathcal{M}_{d}$, i.e., the set of classes of degree $d$ polynomials $f$, all of whose critical points do not escape (equivalently, whose Julia set $J(f)=\operatorname{Bd}(K(f))$ is connected). The Principal Hyperbolic Domain $\mathrm{PHD}_{d}$ of $\mathcal{M}_{d}$ is the set of classes of hyperbolic degree $d$ polynomials with Jordan curve Julia sets; the class $[f]$ of a degree $d$ polynomial $f$ belongs to $\mathrm{PHD}_{d}$ if and only if all critical points of $f$ are in the immediate attracting basin of the same attracting (or super-attracting) fixed point. In [BOPT14 we describe properties of polynomials $f$ such that $[f] \in \overline{\mathrm{PHD}}_{d}$.

Theorem 1.1 (Theorem A [BOPT14]). If $[f] \in \overline{\mathrm{PHD}}_{d}$, then $f$ has a fixed non-repelling point, no repelling periodic cutpoints in $J(f)$, and all its non-repelling periodic points, except at most one fixed point, have multiplier 1. 
Actually, in BOPT14 we claim that all non-repelling periodic cutpoints in the Julia set $J(f)$, except perhaps 0 , have multiplier 1 ; still, literally repeating the same arguments one proves the version of [BOPT14, Theorem A] given by Theorem [1.1. This motivates Definition [1.2, in which we define a special set $\mathrm{CU}$ such that $\overline{\mathrm{PHD}}_{3} \subset \mathrm{CU}$.

Definition 1.2 ([BOPT14]). Let CU be the family of classes of cubic polynomials $f$ with connected $J(f)$ such that $f$ has a non-repelling fixed point, no repelling periodic cutpoints in $J(f)$, and all its nonrepelling periodic points, except at most one fixed point, have multiplier 1. The family CU is called the Main Cubioid.

Let $\mathcal{F}$ be the space of polynomials

$$
f_{\lambda, b}(z)=\lambda z+b z^{2}+z^{3}, \quad \lambda \in \mathbb{C}, \quad b \in \mathbb{C}
$$

parameterized by pairs $(\lambda, b)$ of complex numbers. An affine change of variables reduces any cubic polynomial $f$ to the form $f_{\lambda, b}$. The point 0 is fixed for every $f_{\lambda, b} \in \mathcal{F}$. The set of all polynomials $f \in \mathcal{F}$ such that 0 is non-repelling for $f$ is denoted by $\mathcal{F}_{n r}$. Define the $\lambda$-slice $\mathcal{F}_{\lambda}$ of $\mathcal{F}$ as the space of all polynomials $g \in \mathcal{F}$ with $g^{\prime}(0)=\lambda$. The space $\mathcal{F}$ maps onto the space of classes of all cubic polynomials with a fixed point of multiplier $\lambda$ as a finite branched covering equivalent to the map $b \mapsto a=b^{2}$, i.e., classes of polynomials $f_{\lambda, b} \in \mathcal{F}_{\lambda}$ are in one-to-one correspondence with the values of $a$. Thus, if we talk about, say, points $[f]$ of $\mathcal{M}_{3}$, then it suffices to take $f \in \mathcal{F}_{\lambda}$ for some $\lambda$.

Let $J(f)$ be connected. In Lyu83, MSS83, the notion of $J$-stability was introduced for any holomorphic family of rational functions: a map is $J$-stable if its Julia set admits an equivariant holomorphic motion over a neighborhood of the map in the given family. We say that $f \in \mathcal{F}_{\lambda}$

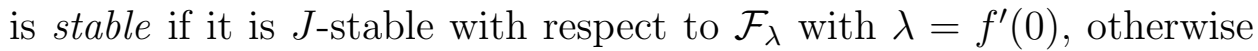
we say that $f$ is unstable. The set $\mathcal{F}_{\lambda}^{s t}$ of all stable polynomials $f \in \mathcal{F}_{\lambda}$ is an open subset of $\mathcal{F}_{\lambda}$. A component of $\mathcal{F}_{\lambda}^{\text {st }}$ is called a $(\lambda$-) stable component or a domain of $(\lambda$-) stability. It is easy to see that, given $\lambda$, the polynomial $f_{\lambda, b}$ has a disconnected Julia set if $|b|$ is sufficiently big. Hence, if $f=f_{\lambda, b}$ is stable and $J(f)$ is connected, then its domain of stability is bounded. For any subset $\mathcal{U} \subset \mathcal{F}$, we let $[\mathcal{U}]$ stand for the set of classes $[f]$ of all polynomials $f \in \mathcal{U}$. If $|\lambda| \leqslant 1$, then we write $\mathcal{P}_{\lambda}$ for the set of all polynomials $f \in \mathcal{F}_{\lambda}$ such that $[f] \in \overline{\mathrm{PHD}}_{3}$.

Theorem 1.3 ([BOPT14]). Suppose that $\mathcal{U}$ is a bounded stable component in $\mathcal{F}_{\lambda},|\lambda| \leqslant 1$, such that $[\mathrm{Bd}(\mathcal{U})] \subset \overline{\mathrm{PHD}}_{3}$. Then $[\mathcal{U}] \subset \mathrm{CU}$. Thus, if $\mathcal{T}_{\lambda}$ is the union of $\mathcal{P}_{\lambda}$ and all $\lambda$-stable components, whose boundaries are contained in $\mathcal{P}_{\lambda}$, then $\left[\mathcal{T}_{\lambda}\right] \subset \mathrm{CU}$. 
For a compactum $X \subset \mathbb{C}$, let the topological hull $\mathrm{TH}(X)$ of $X$ be the complement of the unbounded component of $\mathbb{C} \backslash X$. Lemma 1.4 follows from Corollary 5.3 and gives an equivalent description of $\mathcal{T}_{\lambda}$.

Lemma 1.4. Any component $\mathcal{W}$ of $\mathrm{TH}\left(\mathcal{P}_{\lambda}\right) \backslash \mathcal{P}_{\lambda}$ consists of stable maps. Moreover, $\mathcal{T}_{\lambda}=\mathrm{TH}\left(\mathcal{P}_{\lambda}\right)$ and $\mathrm{TH}\left(\mathcal{P}_{\lambda}\right) \subset \mathrm{CU}$.

In BOPT14a], we study properties of components of $\mathrm{TH}\left(\mathcal{P}_{\lambda}\right) \backslash \mathcal{P}_{\lambda}$, where $|\lambda| \leqslant 1$. Note that these are the same as bounded components of $\mathcal{F}_{\lambda} \backslash \mathcal{P}_{\lambda}$. Let $\mathcal{A}$ be the set of all maps $f_{\lambda, b}$ with $|\lambda|<1$. For each such map $f$, let $A(f)$ be the basin of immediate attraction of 0 . In Section 3, we show that if $f \in \mathcal{F}_{\lambda} \backslash \mathcal{P}_{\lambda}$, then $f$ has two distinct critical points. A critical point $c$ of such an $f$ is said to be principal if there is a neighborhood $\mathcal{U}$ of $f$ in $\mathcal{F}$ and a holomorphic function $\omega_{1}: \mathcal{U} \rightarrow \mathbb{C}$ with $c=\omega_{1}(f)$, and such that, for every $g \in \mathcal{U} \cap \mathcal{A}$, the point $\omega_{1}(g)$ is a unique critical point of $g$ contained in $A(g)$ (the uniqueness of $c$ follows from the fact that $\left.g \notin \overline{\mathrm{PHD}_{3}}\right)$. By Section 3 , the point $\omega_{1}(f)$ is well-defined; denote the other critical point of $f$ by $\omega_{2}(f)$.

Definition 1.5 ([BOPT14a $]$ ). Let $\mathcal{U}$ be a component of $\mathrm{TH}\left(\mathcal{P}_{\lambda}\right) \backslash \mathcal{P}_{\lambda}$. If, for every $f \in \mathcal{U}$, the point 0 belongs to a Siegel domain $U$ of $f$, and there exists an eventual pullback $V$ of $U$ such that $\left.f\right|_{V}$ is two-to-one, we call $\mathcal{U}$ a component of Siegel capture type. If, for every $f \in \mathcal{U}$, the map $f$ has connected Julia set $J(f)$ of positive Lebesgue measure, carries a measurable invariant line field and is such that $\omega_{2}(f) \in J(f)$, then $\mathcal{U}$ is said to be of queer type.

Theorem 1.6 relies upon the tools developed in [BOPT14.

Theorem 1.6 ([BOPT14a] $)$. Suppose that $|\lambda| \leqslant 1$. Then any component of $\mathrm{TH}\left(\mathcal{P}_{\lambda}\right) \backslash \mathcal{P}_{\lambda}$ is either of Siegel capture type or of queer type.

We do not know whether components of Siegel capture type or of queer type exist.

\section{MAin RESUlts}

Let us begin by making a few remarks. If we perturb a cubic polynomial $f$ with a non-repelling fixed point to a polynomial $g$ with an attracting fixed point, then $g$ restricted to the basin of attraction $A(g)$ of that point is either two-to-one or three-to-one. Here, we study polynomials $f$ with a non-repelling fixed point such that $[f] \notin \overline{\mathrm{PHD}}_{3}$, i.e., for all cubic polynomials $g$ sufficiently close to $f$, if $g$ has a fixed attracting point, then $g$ is two-to-one on its basin of immediate attraction. While interesting by itself, this together with [BOPT14] also allows us to learn more about the structure of $\overline{\mathrm{PHD}}_{3}$. 
Next we need a few classic definitions and a major result due to Douady and Hubbard [DH85].

Definition 2.1 ([DH85]). A polynomial-like map (of degree $k$ ) is a holomorphic map $f: U \rightarrow V$ where every point in $V$ has exactly $d$ preimages in $U$ (counted with multiplicities), $U, V$ are open sets isomorphic to the unit disc, and $\bar{U} \subset V$. A polynomial-like map of degree 2 is called a quadratic-like map. The filled Julia set $K(f)$ of $f$ is the set of points in $U$ that never leave $U$ under iteration. The Julia set $J(f)$ of $f$ is the boundary of $K(f)$. Two quadratic-like maps $f: U \rightarrow V$ and $g: U^{\prime} \rightarrow V^{\prime}$ are hybrid equivalent if there is a quasi-conformal map $\varphi: U \rightarrow U^{\prime}$ conjugating $f$ to $g$ such that $\varphi$ is conformal almost everywhere on $K(f)$. The map $\varphi$ is called a straightening map.

It is easy to see that under hybrid equivalence repelling periodic points cannot correspond to non-repelling periodic points.

The following major result is due to Douady and Hubbard; we state it only in the quadratic case.

Theorem 2.2 ([DH85]). Let $f: U \rightarrow V$ be a quadratic-like map. Then $f$ is hybrid equivalent to a quadratic polynomial $P$. Moreover, if $K(f)$ is connected, then $P$ is unique up to (global) conjugation by an affine map.

Say that a cubic polynomial $f \in \mathcal{F}$ is immediately renormalizable if there are Jordan domains $U^{*} \ni 0$ and $V^{*}$ such that $f^{*}=f: U^{*} \rightarrow V^{*}$ is a quadratic-like map (we will use the notation $f^{*}$ at several occasions in the future when we talk about immediately renormalizable maps). If $f \in \mathcal{F}_{n r}$ is immediately renormalizable, then the quadratic-like Julia set $J\left(f^{*}\right)=J^{*}$ is connected. Indeed, $f^{*}$ is hybrid equivalent to a quadratic polynomial $g^{*}$. Since $0 \in J\left(f^{*}\right)$ is a non-repelling $f$-fixed point, it corresponds to a non-repelling fixed point of $g^{*}$. Hence, $J\left(g^{*}\right)$ and $J\left(f^{*}\right)$ are connected, and $g^{*}=z^{2}+c$ with $c \in \overline{\mathrm{PHD}}_{2}$.

Note that, if $[f] \in \overline{\mathrm{PHD}}_{3}$, then $f$ is not immediately renormalizable. Indeed, if $f: U^{*} \rightarrow V^{*}$ is quadratic-like, then $g: U^{*} \rightarrow V^{*}$ is quadraticlike for all $g$ sufficiently close to $f$. If $f, g \in \mathcal{F}_{n r}$ and $0 \in U^{*}$, then the quadratic-like Julia sets of both $f$ and $g$ are connected. Thus, $[g]$ cannot belong to $\mathrm{PHD}_{3}$. If $f \in \mathcal{F}_{n r}$ but $[f] \notin \overline{\mathrm{PHD}}_{3}$, then $f$ is said to be potentially renormalizable. Clearly, the set of all potentially renormalizable polynomials is open in $\mathcal{F}_{n r}$. A connected component of the set of potentially renormalizable polynomials in $\mathcal{F}_{\lambda}$ is called a potentially renormalizable component. For any $f \in \mathcal{F}_{\lambda} \backslash \mathcal{P}_{\lambda}$, let $\mathcal{W}_{f}$ be a potentially renormalizable component containing $f$; the set $\mathcal{W}_{f}$ is 
open in $\mathcal{F}_{\lambda}$, and the set of all potentially renormalizable polynomials is open in $\mathcal{F}$.

We will now discuss the main results of this paper. In Section 3 we introduce special sets $Z(f)$ and $X(f)$ and study them using holomorphic motion. We define a countable set $Z(f)$ of iterated preimages of the principal critical point $\omega_{1}(f)$ as follows: a point $z \in \mathbb{C}$ belongs to $Z(f)$ if there exists an open convex neighborhood $\mathcal{U}_{z}$ of $f$ in $\mathcal{F}$ and a holomorphic function $\zeta: \mathcal{U}_{z} \rightarrow \mathbb{C}$ so that $(1) \zeta(f)=z$, (2) $g^{\circ n}(\zeta(g))=\omega_{1}(g)$ for all $g \in \mathcal{U}_{z}$ and for some $n \geqslant 0$ independent of $g$, and (3) $\zeta(g) \in A(g)$ for all $g \in \mathcal{U}_{z} \cap \mathcal{A}$. Then we study properties of $Z(f)$, define sets $Z_{n}(f)$ as the subsets of $Z(f)$ consisting of all preimages of $\omega_{1}(f)$ mapped to $\omega_{1}(f)$ in $n$ steps, and define the set $X(f)$ as the limit of the sets $Z_{n}(f)$, i.e., $X(f)=\bigcap_{m \geqslant 0} \overline{\bigcup_{n \geqslant m} Z_{n}(f)}$. The main result of Section 3 is Theorem A.

Theorem A. Suppose that $f \in \mathcal{W}_{f}$, where $\mathcal{W}_{f}$ is a potentially renormalizable component of $\mathcal{F}_{\lambda} \backslash \mathcal{P}_{\lambda}$. Then there is an equivariant holomorphic motion $\bar{\mu}: X(f) \times \mathcal{W}_{f} \rightarrow \mathbb{C}$. The set $X(f)$ is a forward invariant subset of $J(f)$. It contains no neutral periodic points different from 0 . Every point of $X(f)$ has at least two preimages in $X(f)$ counting multiplicities.

In Section 4 we study polynomial-like maps. The main result of Section 4 is Theorem B.

Theorem B. Let $P: \mathbb{C} \rightarrow \mathbb{C}$ be a polynomial, and $Y \subset \mathbb{C}$ be a non-separating $P$-invariant continuum. The following assertions are equivalent:

(1) the set $Y$ is the filled Julia set of some polynomial-like map $P: U^{*} \rightarrow V^{*}$ of degree $k$,

(2) $Y$ is a component of the set $P^{-1}(P(Y))$ and, for every attracting or parabolic point $y$ of $P$ in $Y$, the attracting basin of $y$ or the union of all parabolic domains at $y$ is a subset of $Y$.

The proof of Theorem B uses some ideas communicated by M. Lyubich to the fourth named author.

Finally, in Section 5 we prove Theorem C, in which we show that potentially renormalizable polynomials are immediately renormalizable depending on transparent assumptions about their dynamics.

Theorem C. Let $|\lambda| \leqslant 1$ and $f \in \mathcal{F}_{\lambda} \backslash \mathcal{P}_{\lambda}$. Then one of the following holds:

(1) $f$ belongs to the unbounded component of $\mathcal{F}_{\lambda} \backslash \mathcal{P}_{\lambda}$ and $f$ is immediately renormalizable; 
(2) $f$ belongs to a bounded component of $\mathcal{F}_{\lambda} \backslash \mathcal{P}_{\lambda}$ of Siegel capture type and $f$ is not immediately renormalizable;

(3) $f$ belongs to a bounded component of $\mathcal{F}_{\lambda} \backslash \mathcal{P}_{\lambda}$ of queer type, and there are two possibilities: (a) the entire orbit of $\omega_{2}(f)$ is disjoint from $X(f)$ and $f$ is immediately renormalizable, or (b) $\omega_{2}(f) \in X(f)$ and $f$ is not immediately renormalizable.

If $f$ is immediately renormalizable, the corresponding quadratic-like map is hybrid equivalent to a quadratic polynomial $z^{2}+c$ with $c \in \overline{\mathrm{PHD}}_{2}$ or, equivalently, to the polynomial $\lambda z+z^{2}$.

We do not know whether any of the options mentioned in parts (2) and (3) realizes. The authors are indebted to the referee, whose suggestions led to the proof of part (3) of Theorem C.

There is no loss of generality in that we consider only perturbations of $f$ in $\mathcal{F}$ : instead, we could consider small perturbations $g$ of $f$ such that, arbitrarily close to 0 , the map $g$ has an attracting fixed point. Theorem A generalizes some results from [BuHe01, Zak99].

Notation and Preliminaries: we write $\bar{A}$ for the closure of a subset $A$ of a topological space and $\operatorname{Bd}(A)$ for the boundary of $A$; the $n$-th iterate of a map $f$ is denoted by $f^{\circ n}$. Let $\mathbb{C}$ stand for the complex plane, $\mathbb{C}^{*}$ for the Riemann sphere, $\mathbb{D}$ for the open unit disk consisting of all complex numbers $z$ with $|z|<1$, and $\mathbb{S}=\operatorname{Bd}(\mathbb{D})$ for the unit circle, which is identified with $\mathbb{R} / \mathbb{Z}$. The $d$-tupling map of the unit circle is denoted by $\sigma_{d}$. We assume knowledge of basic notions from complex dynamics, such as Green function, dynamic rays (of specific argument), Böttcher coordinate, Fatou domain, repelling, attracting, neutral periodic points, parabolic, Siegel, Cremer periodic points etc (see, e.g., [McM94]).

\section{Potentially Renormalizable polynomials}

Throughout Section 3, we consider a potentially renormalizable cubic polynomial $f$. We want to see when $f$ is immediately renormalizable. Below we outline our strategy; to motivate our approach, assume for the moment that $f$ is already immediately renormalizable. The main idea is to observe that some points and sets related to the quadratic-like restriction $f^{*}$ (including $J^{*}=J\left(f^{*}\right)$ ) can actually be defined independently of the fact that $f$ is immediately renormalizable. This allows us to define them for all potentially renormalizable maps $f$; in other words, we define a potential quadratic-like Julia set and then prove that in some cases the potential of being a quadratic-like Julia set is realized.

Recall that $\mathcal{A}$ is the set of all cubic polynomials $g \in \mathcal{F}$ with $\left|g^{\prime}(0)\right|<$ 1 , and, for $g \in \mathcal{A}$, we write $A(g)$ for the immediate basin of attraction 
of 0 with respect to $g$. If $g \in \mathcal{A}$ is such that there is a unique critical point in $A(g)$, we let $\omega_{1}(g)$ be this critical point and let $\omega_{2}(g)$ be the other critical point of $g$. As a tool, we consistently approximate potentially renormalizable maps $f$ by polynomials from $\mathcal{A}$. If a potentially renormalizable map $f$ belongs to $\mathcal{A}$, then $f$ itself serves as its own approximation. Since $f$ is potentially renormalizable, there is a neighborhood of $f$ in $\mathcal{F}$, in which there is no polynomial $g \in \mathcal{A}$ with $[g] \in \mathrm{PHD}_{3}$.

First we define the critical points $\omega_{1}(f)$ and $\omega_{2}(f)$ for all potentially renormalizable maps, and show that these points depend holomorphically on $f \in \mathcal{F}_{\lambda} \backslash \mathcal{P}_{\lambda}$. We next consider a countable set $Z(f)$ of iterated $f$-preimages of $\omega_{1}(f)$, each of which depends holomorphically on $f \in \mathcal{F}_{\lambda} \backslash \mathcal{P}_{\lambda}$. For $g \in \mathcal{A}$ with a unique critical point $\omega_{1}(g) \in A(g)$, the set $Z(g)$ is the set of all iterated $g$-preimages of $\omega_{1}(g)$ contained in $A(g)$. Finally, the potential quadratic-like Julia set of $f$ can be defined as the set of all non-isolated points in $\bar{Z}(f)$. We will show that this set moves holomorphically with $f$.

\subsection{The principal critical point of $f$. Fix $f \in \mathcal{F}_{\lambda} \backslash \mathcal{P}_{\lambda}$ as above.}

Lemma 3.1. The polynomial $f$ has two distinct critical points.

Proof. Assume that $\omega(f)$ is the only critical point of $f$ (then it has multiplicity two). Let $\mathcal{C}$ be the space of all polynomials $g \in \mathcal{F}$ with a multiple critical point $\omega(g)$. This is an algebraic curve in $\mathcal{F}$ passing through $f$ and given by the formula $g_{a}(z)=\frac{a^{2}}{3} z+a z^{2}+z^{3}, a \in \mathbb{C}$. This implies that there are polynomials $g \in \mathcal{C}$ arbitrarily close to $f$, for which $\left|g^{\prime}(0)\right|<1$. The class of any such polynomial $g$ belongs to $\mathrm{PHD}_{3}$ as the immediate basin of 0 with respect to $g$ must contain the multiple critical point $\omega(g)$, contradicting our assumption on $f$.

By Lemma 3.1, there are two critical points of $f$. Recall, that a critical point $c$ of $f$ is said to be principal if there is a neighborhood $\mathcal{U}$ of $f$ in $\mathcal{F}$ and a holomorphic function $\omega_{1}: \mathcal{U} \rightarrow \mathbb{C}$ defined on this neighborhood such that $c=\omega_{1}(f)$, and, for every $g \in \mathcal{U} \cap \mathcal{A}$, the point $\omega_{1}(g)$ is the critical point of $g$ contained in $A(g)$.

Theorem 3.2. There exists a unique principal critical point of $f$.

Proof. By Lemma 3.1, the two critical points of $f$ are different. Then there are two holomorphic functions, $\omega_{1}$ and $\omega_{2}$, defined on a convex neighborhood $\mathcal{U}$ of $f$ in $\mathcal{F}$, such that $\omega_{1}(g)$ and $\omega_{2}(g)$ are the critical points of $g$ for all $g \in \mathcal{U}$. Suppose that neither $\omega_{1}(f)$, nor $\omega_{2}(f)$ is principal. Then, arbitrarily close to $f$, there are cubic polynomials $g_{1} \in \mathcal{A} \cap \mathcal{U}$ and $g_{2} \in \mathcal{A} \cap \mathcal{U}$ with $\omega_{2}\left(g_{1}\right) \notin A\left(g_{1}\right)$ and $\omega_{1}\left(g_{2}\right) \notin A\left(g_{2}\right)$. 
Since $A\left(g_{i}\right)$ contains a critical point for $i=1,2$, we must have that $\omega_{i}\left(g_{i}\right) \in A\left(g_{i}\right)$.

The set $\mathcal{A}$ is convex. Therefore, the intersection $\mathcal{U} \cap \mathcal{A}$ is also convex, hence connected. Let $\mathcal{O}_{i}, i=1,2$, be the subset of $\mathcal{U} \cap \mathcal{A}$ consisting of all polynomials $g$ with $\omega_{i}(g) \in A(g)$. By the preceding paragraph, $g_{1} \in \mathcal{O}_{1}$ and $g_{2} \in \mathcal{O}_{2}$. We claim that $\mathcal{O}_{i}$ is open. Indeed, if $g \in \mathcal{O}_{i}$, then there exists a Jordan disk $U \subset A(g)$ with $g(U)$ compactly contained in $U$, and $\omega_{i}(g) \in U$. If $\tilde{g} \in \mathcal{U} \cap \mathcal{A}$ is sufficiently close to $g$, then $\tilde{g}(U)$ is still compactly contained in $U$, and $\omega_{i}(\tilde{g})$ is still in $U$, by continuity. It follows that $U \subset A(\tilde{g})$, in particular, $\omega_{i}(\tilde{g}) \in A(\tilde{g})$. Thus, $\mathcal{O}_{i}$ is open. Since $\mathcal{O}_{1}, \mathcal{O}_{2}$ are open and non-empty, the set $\mathcal{U} \cap \mathcal{A}$ is connected, and

$$
\mathcal{U} \cap \mathcal{A}=\mathcal{O}_{1} \cup \mathcal{O}_{2}
$$

the intersection $\mathcal{O}_{1} \cap \mathcal{O}_{2}$ is nonempty. Note that $\mathcal{O}_{1} \cap \mathcal{O}_{2}$ consists of polynomials, whose classes are in $\mathrm{PHD}_{3}$. Since $\mathcal{U}$ can be chosen arbitrarily small, it follows that $f$ can be approximated by maps $g \in \mathcal{A}$ with $[g] \in \mathrm{PHD}_{3}$, a contradiction.

The existence of a principal critical point of $f$ is thus proved. The uniqueness follows immediately from the fact that $f \notin \overline{\mathrm{PHD}_{3}}$.

Denote by $\omega_{1}(f)$ the principal critical point of $f$ and by $\omega_{2}(f)$ the other critical point of $f$. For $g \in \mathcal{F}_{n r}$ sufficiently close to $f$, the point $\omega_{1}(g)$ is a holomorphic function of $g$.

Lemma 3.3. Let $m$ and $n$ be two non-negative integers. Then for each slice $\lambda,|\lambda| \leqslant 1$ the function $\varphi_{m, n}(f)=f^{m}\left(\omega_{1}(f)\right)-f^{n}\left(\omega_{2}(f)\right)$ is not a constant on the slice $\mathcal{F}_{\lambda}$.

Proof. Suppose otherwise and let $\varphi_{m, n}(f)=s$. Observe that there exists $M$ such that for each $g \in \mathcal{F}_{n r}$ with $\left|g^{\prime}(0)\right|<1$ the set $A(g)$ is contained in the disk $D$ of radius $M$ centered at 0 . Since arbitrarily close to any $f \in \mathcal{F}_{n r}$ there are maps $g \in \mathcal{F}_{n r}$ with $\left|g^{\prime}(0)\right|<1$, it follows that the $f$-orbit of $\omega_{1}(f)$ is contained in $D$ for any $f \in \mathcal{F}_{\lambda}$. Since by the assumption $\varphi_{m, n}(f)=s$ it follows that the $f$-orbit of $\omega_{2}(f)$ is contained in the disk $\widehat{D}$ of radius $M+|s|$ centered at 0 . Thus, in this case on the entire $\mathcal{F}_{\lambda}$ both critical points are non-escaping, a contradiction.

3.2. Holomorphic motion. Let $\Lambda$ be a Riemann surface, and $Z \subset \mathbb{C}^{*}$ any (!) subset. A holomorphic motion of the set $Z$ is a map $\mu: Z \times \Lambda \rightarrow$ $\mathbb{C}^{*}$ with the following properties:

- for every $z \in Z$, the map $\mu(z, \cdot):\{z\} \times \Lambda \rightarrow \mathbb{C}^{*}$ is holomorphic;

- for $z \neq z^{\prime}$ and every $\nu \in \Lambda$, we have $\mu(z, \nu) \neq \mu\left(z^{\prime}, \nu\right)$;

- there is a point $\nu_{0}$ such that $\mu\left(z, \nu_{0}\right)=z$ for all $z \in Z$. 
We will use the following crucial $\lambda$-lemma of Mañé, Sad and Sullivan [MSS83]: a holomorphic motion of a set $Z$ extends to a unique holomorphic motion of the closure $\bar{Z}$; moreover, this extension is a continuous function in two variables such that, for every $\nu \in \Lambda$, the $\operatorname{map} \varphi: \bar{Z} \rightarrow \mathbb{C}^{*}$ defined as $\varphi(z)=\mu(z, \nu)$ is quasi-symmetric. There have been useful generalizations of this result, but we will only need the original version. It is worth mentioning here that the simplest version of the $\lambda$-lemma (extension to the closure) appeared also in [Lyu83.

We will now define a countable set $Z(f)$ of iterated preimages of the principal critical point $\omega_{1}(f)$. By definition, a point $z \in \mathbb{C}$ belongs to $Z(f)$ if there exists an open convex neighborhood $\mathcal{U}_{z}$ of $f$ in $\mathcal{F}$ and a holomorphic function $\zeta: \mathcal{U}_{z} \rightarrow \mathbb{C}$ with the following properties:

- $\zeta(f)=z$

- we have $g^{\text {on }}(\zeta(g))=\omega_{1}(g)$ for all $g \in \mathcal{U}_{z}$ and for some $n \geqslant 0$ independent of $g$;

- we have $\zeta(g) \in A(g)$ for all $g \in \mathcal{U}_{z} \cap \mathcal{A}$.

A holomorphic function $\zeta: \mathcal{U}_{z} \rightarrow \mathbb{C}$ like above is called a deformation of $z \in Z(f)$. As it is always clear what kind of deformation we consider, in what follows we will suppress the subscript in the notation for $\mathcal{U}$.

For any $f \in \mathcal{F}_{\lambda} \backslash \mathcal{P}_{\lambda}$, let $\mathcal{W}_{f}$ be a potentially renormalizable component containing $f$; clearly, $\mathcal{W}_{f}$ is open.

Lemma 3.4. Let $f$ be as above.

(1) The critical point $\omega_{1}(f)$ is not eventually mapped to $\omega_{2}(f)$.

(2) The set $Z(f)$ contains no critical values of $f$.

Proof. Suppose first that $\omega_{1}(f)$ is eventually mapped to $\omega_{2}(f)$, say, $f^{\circ m}\left(\omega_{1}(f)\right)=\omega_{2}(f)$, and the number $m$ is the minimal positive integer with this property. Consider the set $\mathcal{C}$ of all $g \in \mathcal{U}$ such that $g^{\circ m}\left(\omega_{1}(g)\right)=\omega_{2}(g)$. This set is an open part (not necessarily connected) of an algebraic curve. The function $g \mapsto g^{\prime}(0)$ is a complex analytic function on $\mathcal{C}$. By Lemma 3.3 this function is not a constant. Since the value of this function at $f$ lies in $\overline{\mathbb{D}}$, there are maps $g \in \mathcal{C}$ arbitrarily close to $f$ such that $\left|g^{\prime}(0)\right|<1$. The class of any such $g$ must belong to $\mathrm{PHD}_{3}$. Indeed, the attracting basin $A(g)$ must contain the principal critical point $\omega_{1}(g)$, by definition of the principal critical point. Since $\omega_{1}(g)$ is eventually mapped to $\omega_{2}(g)$, the critical point $\omega_{2}(g)$ is also contained in $A(g)$. We arrive at a contradiction with our assumption on $f$.

Suppose now that $v \in Z(f)$ is a critical value. Let $\zeta: \mathcal{U} \rightarrow \mathbb{C}$ be a deformation of $v$. Consider the set $\mathcal{C}$ of all $g \in \mathcal{U}$ such that $\zeta(g)$ is a critical value. This set is a part of an algebraic curve. Take a sequence 
$g_{n} \in \mathcal{C} \cap \mathcal{A}$ that converges to $f$. Since $\zeta\left(g_{n}\right) \in A\left(g_{n}\right)$ is a critical value with at least two $g_{n}$-preimages in $A\left(g_{n}\right)$, counting multiplicities, the set $A\left(g_{n}\right)$ must contain a critical point $d_{n}$ with $g_{n}\left(d_{n}\right)=\zeta\left(g_{n}\right)$. The fact that $\omega_{1}\left(g_{n}\right)$ is not periodic implies that $d_{n} \neq \omega_{1}\left(g_{n}\right)$. Thus, both critical points of $g_{n}$ are contained in $A\left(g_{n}\right)$, and so $\left[g_{n}\right] \in \mathrm{PHD}_{3}$. We again arrive at a contradiction with our assumption on $f$.

Lemma 3.5. For every $z \in Z(f)$, there are exactly two points of $Z(f)$ that are mapped to $z$ under $f$.

Proof. The proof is similar to that of Theorem 3.2. Let $\zeta: \mathcal{U} \rightarrow \mathbb{C}$ be a deformation of $z$. Since the set $Z(f)$ cannot contain a critical value of $f$, there are three holomorphic functions $\zeta_{1}, \zeta_{2}, \zeta_{3}$ defined on $\mathcal{U}$ and such that $g\left(\zeta_{i}(g)\right)=\zeta(g)$ (we may need to pass to a smaller neighborhood $\mathcal{U}$ to arrange this).

The intersection $\mathcal{U} \cap \mathcal{A}$ is convex, hence connected. For any 2-element subset $\{i, j\} \subset\{1,2,3\}$, define a subset $\mathcal{O}_{i j} \subset \mathcal{U} \cap \mathcal{A}$ as the set of all polynomials $g \in \mathcal{U} \cap \mathcal{A}$ such that $\zeta_{i}(g) \in A(g)$ and $\zeta_{j}(g) \in A(g)$. All three sets $\mathcal{O}_{12}, \mathcal{O}_{23}$ and $\mathcal{O}_{13}$ are open (cf. the proof of Theorem 3.2). On the other hand, we have

$$
\mathcal{A} \cap \mathcal{U}=\mathcal{O}_{12} \cup \mathcal{O}_{23} \cup \mathcal{O}_{13}
$$

Hence either only one of the sets $\mathcal{O}_{i j}$ is nonempty, or at least two of the sets $\mathcal{O}_{i j}$ intersect. In the latter case, $\zeta_{i}(g) \in A(g)$ for some $g \in \mathcal{A} \cap \mathcal{U}$ and all $i=1,2$, 3. It follows that $[g] \in \mathrm{PHD}_{3}$. Since the neighborhood $\mathcal{U}$ can be chosen to be arbitrarily small, it follows that $f$ can be approximated by polynomials in $\mathcal{A}$, whose classes are in $\mathrm{PHD}_{3}$, a contradiction. The contradiction shows that only one of the sets $\mathcal{O}_{i j}$ is nonempty, for a suitable choice of the neighborhood $\mathcal{U}$. Assume that $i=1$ and $j=2$; then $\zeta_{1}(f), \zeta_{2}(f) \in Z(f)$ but $\zeta_{3}(f) \notin Z(f)$.

The proof of Lemma 3.5 implies a stronger claim below.

Corollary 3.6. Let $\zeta_{i}$ be holomorphic functions introduced in the proof of Lemma 3.5. Suppose that $\zeta_{1}(f), \zeta_{2}(f) \in Z(f)$. Then there is a neighborhood $\mathcal{U}$ of $f$ in $\mathcal{F}$ such that $\zeta_{3}(g) \notin A(g)$ for all $g \in \mathcal{U} \cap \mathcal{A}$.

We will need the following lemma.

Lemma 3.7. If $|\lambda|<1$, then the set of all polynomials $f \in \mathcal{F}_{\lambda}$ such that both critical points of $f$ belong to the immediate basin of attraction of 0 is an open simply connected domain. Moreover, if $|\lambda| \leqslant 1$, then the set $\mathcal{P}_{\lambda}$ is connected.

Proof. Suppose that $|\lambda|<1$. Consider the branched covering map $\Psi$ of $\mathbb{C}$ by $\mathcal{F}_{\lambda}$ sending $f_{\lambda, b}$ to $b^{2}$. The fibers of this map are exactly affine 
conjugacy classes in $\mathcal{F}_{\lambda}$. By parts b2) and c) of [PeTa09, Theorem $A^{\prime}$, the $\Psi$-image of $\mathcal{P}_{\lambda}$ is a closed Jordan disk (see also [PiTa04]). The set $\mathcal{P}_{\lambda}$ is therefore a disk or a pair of disks depending on whether or not the polynomial $f=f_{\lambda, 0}(z)=\lambda z+z^{3}$ belongs to $\mathcal{P}_{\lambda}$. Observe that $f$ is odd, therefore, critical points are antipodal and the basin $A(f)$ of immediate attraction of 0 is centrally symmetric with respect to the origin. Since at least one of the critical points must belong to $A(f)$, then both do which implies that $f \in \mathcal{P}_{\lambda}$ as desired.

Suppose now that $|\lambda| \leqslant 1$. By [PeTa09], the set $\mathcal{P}_{\lambda^{\prime}}$ depends continuously on $\lambda^{\prime}$. The set $\mathcal{P}_{\lambda}$ is the upper limit of $\mathcal{P}_{\lambda^{\prime}}$ as $\lambda^{\prime} \rightarrow \lambda$. Since the upper limit of a continuous family of connected sets (over a locally connected base) is connected, the set $\mathcal{P}_{\lambda}$ is connected.

Lemma 3.7 was included at the suggestion of the referee; we are indebted to him/her for this suggestion.

Proposition 3.8. For every $z \in Z(f)$, there is a holomorphic function $\zeta: \mathcal{W}_{f} \rightarrow \mathbb{C}$ such that $\zeta(h) \in Z(h)$ for all $h \in \mathcal{W}_{f}$ and $\zeta(f)=z$.

Proof. The function $\zeta$ with these properties is defined at least on some open neighborhood of $f$ in $\mathcal{W}_{f}$, by definition of the set $Z(f)$. Assume by induction that the statement of the proposition holds for the point $f(z)$, i.e., there is a holomorphic function $\eta: \mathcal{W}_{f} \rightarrow \mathbb{C}$ such that $\eta(h) \in Z(h)$ for all $h \in \mathcal{W}_{f}$ and $\eta(f)=f(z)$. It follows that there is an integer $n$ such that $h^{\circ(n-1)}(\eta(h))=\omega_{1}(h)$ for all $h \in \mathcal{W}_{f}$. Consider the multivalued analytic function $h \mapsto h^{-1}(\eta(h))$. If this function has no branch points in $\mathcal{W}_{f}$, then we can define the holomorphic function $\zeta$ as the branch of this function such that $\zeta(f)=z$. Suppose that there is a branch point $h_{0}$ of the multivalued function $h \mapsto h^{-1}(\eta(h))$. Then the point $\eta\left(h_{0}\right)$ is a critical value of $h_{0}$, a contradiction with Lemma 3.4 .

By Lemma 3.7, the set $\mathcal{W}_{f}$ is simply connected, therefore, a multivalued analytic function without branching is necessarily a disjoint union of single valued branches. Thus, there is a holomorphic function $\zeta: \mathcal{W}_{f} \rightarrow \mathbb{C}$ with $h(\zeta(h))=\eta(h)$, and $\zeta(f)=z$. Moreover, $\zeta(h) \in Z(h)$ for all $h \in \mathcal{W}_{f}$ sufficiently close to $f$. It suffices to prove that $\zeta(h) \in Z(h)$ for all $h \in \mathcal{W}_{f}$. To this end, we will prove that the set of polynomials $h \in \mathcal{W}_{f}$ such that $\zeta(h) \in Z(h)$ is open and closed in $\mathcal{W}_{f}$. The openness is obvious. Consider a sequence $h_{n} \in \mathcal{W}_{f}$ converging to some polynomial $h \in \mathcal{W}_{f}$, and suppose that $\zeta\left(h_{n}\right) \in Z\left(h_{n}\right)$ but $\zeta(h) \notin Z(h)$. Therefore, there are two other holomorphic functions $\zeta_{1}$, $\zeta_{2}$ defined on some neighborhood of $h$ such that $\zeta_{i}(h) \in Z(h), i=1$, 2. It follows that $\zeta_{i}\left(h_{n}\right) \in Z\left(h_{n}\right)$ for sufficiently large $n$. But then all 
three points $\zeta_{1}\left(h_{n}\right), \zeta_{2}\left(h_{n}\right)$ and $\zeta\left(h_{n}\right)$ are preimages of $\eta\left(h_{n}\right)$ in $Z\left(h_{n}\right)$. This contradicts Lemma 3.5.

Proposition 3.8 and Lemma 3.4 imply the following theorem.

Theorem 3.9. There exists a holomorphic motion $\mu: Z(f) \times \mathcal{W}_{f} \rightarrow \mathbb{C}$ that is equivariant in the sense that for every $h \in \mathcal{W}_{f}$, and for every $z \in Z(f) \backslash\left\{\omega_{1}(f)\right\}$, we have $h(\mu(z, h))=\mu(f(z), h)$.

By the $\lambda$-lemma, the holomorphic motion $\mu$ gives rise to the holomorphic motion $\bar{\mu}: \overline{Z(f)} \times \mathcal{W}_{f} \rightarrow \mathbb{C}$. Since $\mu$ is equivariant, the holomorphic motion $\bar{\mu}$ is equivariant too.

3.3. The set $X(f)$. Let $Z_{n}(f)$ be the subset of $Z(f)$ consisting of all preimages of $\omega_{1}(f)$ mapped to $\omega_{1}(f)$ in $n$ steps, in other words, $z \in Z_{n}(f)$ if $f^{\circ n}(z)=\omega_{1}(f)$. Define the set $X(f)$ as the limit of the sets $Z_{n}(f)$, i.e.,

$$
X(f)=\bigcap_{m \geqslant 0} \overline{\bigcup_{n \geqslant m} Z_{n}(f) .}
$$

It is easy to see that $X(f)$ coincides with the set of all non-isolated points in $\overline{Z(f)}$. Theorem 3.9 and the $\lambda$-lemma imply that the sets $X(h)$ move holomorphically for $h \in \mathcal{W}_{f}$. Clearly, $X(h)$ is forward invariant under $h$.

Let $P$ be a polynomial of degree $d$. Then $P$ on a small neighborhood of any point $t$ is $k$-to- 1 (at regular points $t$, we have $k=1$, and at critical points $k>1$ ); the number $k$ is called the multiplicity of $t$.

Lemma 3.10. Let $h \in \mathcal{W}_{f}$. Then the set $X(h)$ is a subset of the Julia set $J(h)$, and every point $x \in X(h)$ has at least two preimages in $X(h)$, counting multiplicities.

Proof. The set $X(h)$ is contained in the accumulation set of the backward orbit of $\omega_{1}(h)$. The backward orbit of a point can accumulate in the Fatou set only if the point lies in a Siegel disk. However $\omega_{1}(h)$ cannot lie in a Siegel disk as a Siegel disk contains no critical points. The second claim follows from Lemma 3.5.

Recall that, by the $\lambda$-lemma, $\bar{\mu}: \overline{Z(f)} \times \mathcal{W}_{f} \rightarrow \mathbb{C}$ is continuous. In particular, if a sequence $z_{n} \in \overline{Z(f)}$ converges to $z \in \overline{Z(f)}$, then $\mu\left(z_{n}, h\right)$ converges to $\mu(z, h)$, for every $h \in \Lambda$.

Lemma 3.11. The set $X(f)$ contains no neutral periodic points different from 0 . 
Proof. Let $X(f)$ contain a periodic neutral point $x \neq 0$ of minimal period $k$. Since the holomorphic motion $\bar{\mu}$ is equivariant, $\bar{\mu}(x, h)=$ $\bar{\mu}\left(f^{\circ r}(x), h\right)=h^{\circ r}(\bar{\mu}(x, h))$ for every $r$. This proves that $\bar{\mu}(x, h)=x(h)$ is a periodic point of $h$ of period $k$, for every $h \in \mathcal{W}_{f}$.

The holomorphic function $h \mapsto\left(h^{\circ k}\right)^{\prime}(x(h))$ is non-constant on the multiplier slice $\mathcal{F}_{\lambda}$. Indeed, the slice $\mathcal{F}_{\lambda}$ contains polynomials with disconnected Julia sets, and such polynomials cannot have non-repelling periodic points different from 0 , by the Fatou-Shishikura inequality. It follows that $x(h)$ is an attracting periodic point with respect to $h$, for some polynomials $h$ in arbitrarily small neighborhood of $f$, a contradiction to Lemma 3.10.

Theorem A explicitly summarizes the results of this section.

Theorem A. Suppose that $f \in \mathcal{W}_{f}$ where $\mathcal{W}_{f}$ is a potentially renormalizable component of $\mathcal{F}_{\lambda} \backslash \mathcal{P}_{\lambda}$. Then there is an equivariant holomorphic motion $\bar{\mu}: X(f) \times \mathcal{W}_{f} \rightarrow \mathbb{C}$. The set $X(f)$ is a forward invariant subset of $J(f)$. It contains no neutral periodic points different from 0. Every point of $X(f)$ has at least two preimages in $X(f)$ counting multiplicities.

Finally in this section we state Lemma 3.12 .

Lemma 3.12. Suppose that $f \in \mathcal{F}_{n r}$ is immediately renormalizable and $J^{*}(f)$ is its quadratic-like Julia set. Then $J^{*}(f)=X(f)$ and the principal critical point $\omega_{1}(f)$ belongs to $\mathrm{TH}(X(f))$.

Proof. Left to the reader.

In the rest of the paper, we adopt the following approach. First we establish several types of conditions on $X(f)$ and the holomorphic motion $\bar{\mu}$ sufficient for $f$ being immediately renormalizable; the set $X(f)$ plays here the role of a potential quadratic-like Julia set. Then we verify that these conditions are fulfilled for various cubic polynomials. In the end, this will lead to the proofs of our results.

\section{Properties of POLYNOMIAL-LIKE MAPS}

In this section, we prove a criterion for a polynomial $P$ of any degree to have a polynomial-like restriction. Recall that, for any map $F$, by an $F$-invariant set, we mean a set $A$ such that $F(A) \subset A$ but not necessarily $F(A)=A$.

We start with some purely topological considerations. Consider a compactum $T \subset \mathbb{C}^{*}$ and a branch covering $P: \mathbb{C}^{*} \rightarrow \mathbb{C}^{*}$. Let $\nu_{T}(z)$ be the number of all $P$-preimages of $z$ in $T$ counted with multiplicities. Then there exists a neighborhood $V$ of $z$ and $r$ pullbacks $W_{1}, \ldots, W_{r}$ 
of $V$ each containing exactly one point of the set $P^{-1}(z) \cap T$ and such that the sum of degrees of $P$ restricted on $W_{1}, \ldots, W_{r}$ is $\nu_{T}(z)$. If a point $x \in P^{-1}(z) \cap T$ is not critical and belongs to $W_{i}$, the map $\left.P\right|_{W_{i}}$ is a homeomorphism onto image. If a point $x \in P^{-1}(z) \cap T \cap W_{i}$ is critical, then it is the unique critical point of $P$ in $W_{i}$.

Set $\widehat{W}=\cup_{i=1}^{r} W_{i}$. By compactness, the $P$-image of $T \backslash \widehat{W}$ is positively distant from $z$. Hence, for some smaller neighborhood $V^{\prime} \subset V$ of $z$, all preimages of any point $z^{\prime} \in V^{\prime}$ in $T \cap P^{-1}\left(V^{\prime}\right)$ belong to $\widehat{W}$ and the entire preimage of $V^{\prime}$ in $T$ breaks down into $r$ pieces contained in $W_{1}, \ldots, W_{r}$. From now on let us call such $V^{\prime}$ a $(T-)$ suitable neighborhood of $z^{\prime}$. Since any point $y \in V$ has exactly $\nu_{T}(z)$ preimages in $\widehat{W}$ (not necessarily in $T$ ), the value of $\nu_{T}$ can only drop at points $z^{\prime} \in V^{\prime}$, and $\nu_{T}$ is upper-semicontinuous.

Let $\left.\nu_{T}\right|_{P(T)}$ be continuous at $z$. Choose a suitable neighborhood $V$ of $z$, on which $\left.\nu_{T}\right|_{P(T)}$ is constant. Then, for every point $y \in V \cap P(T)$, the set $P^{-1}(y) \cap T$ of its preimages in $T$ consists exactly of all its preimages in $\widehat{W}$. Indeed, by the previous paragraph, if $y \in V$, then it has $\nu_{T}(z)$ preimages in $\widehat{W}$. Together with the fact that $\nu_{T}(y)=\nu_{T}(z)$ this implies our claim. Hence if $x \in P(T)$ is a point of continuity of $\left.\nu_{T}\right|_{P(T)}$, then $x$ has a neighborhood $V$ such that in the corresponding open set $\widehat{W}$ points from $T$ and not from $T$ cannot have the same image. If $\left.\nu_{T}\right|_{P(T)}$ is continuous at all points, it follows that there exists a neighborhood $U$ of $T$ such that for any $z \in U \backslash T$ we have $P(z) \notin P(T)$. It is equally easy to show that, conversely, if $\left.\nu_{T}\right|_{P(T)}$ is discontinuous at $z$, then there is a preimage of $z$ in $T$, in whose arbitrarily small neighborhood "collisions" between a point from $T$ and a point not from $T$ take place. All this is summarized in Lemma 4.1.

Lemma 4.1. Suppose that $T \subset \mathbb{C}^{*}$ is a compact set and $P: \mathbb{C}^{*} \rightarrow \mathbb{C}^{*}$ is a branch covering. Then the following two properties are equivalent.

(1) The function $\nu_{T}$ is continuous on $P(T)$.

(2) There exists a neighborhood $U$ of $T$ such that for any $z \in U \backslash T$ we have $P(z) \notin P(T)$.

If $T$ is connected, these conditions are equivalent to the following:

(3) $T$ is a component of $P^{-1}(P(T))$.

(4) $\nu_{T}$ is a constant on $T$.

Proof. By the arguments right before Lemma 4.1, claims (1) and (2) are equivalent. Assume now that $T$ is connected. Then (1) and (4) are equivalent, and (2) implies (3). Suppose that (3) holds. Then there is a neighborhood $U$ of $T$ that does not intersect other components of $P^{-1}(P(T))$. It follows for every $z \in U \backslash T$ we have $P(z) \notin P(T)$. 
There is a useful sufficient condition for (3) in the polynomial case.

Lemma 4.2. Suppose that $T$ is a continuum and $P$ is a polynomial. Set $m=1+\sum\left(d_{c}-1\right)$, where the sum is taken over all critical points $c$ of $P$ in $T$, and $d_{c}$ is the multiplicity of the point c. If $(1) P(c) \notin \mathrm{TH}(P(T))$ for any critical point $c \notin \mathrm{TH}(T)$, (2) there are no critical points in $\mathrm{TH}(T) \backslash T$, and (3) for each point $x \in P(T)$ we have $\nu_{T}(x) \geqslant m$, then $T$ is a component of the set $P^{-1}(P(T))$ and $\mathrm{TH}(T)$ is a component of the set $P^{-1}(P(\mathrm{TH}(T))$ ) (in particular, $\mathrm{TH}(P(T))=P(\mathrm{TH}(T))$ ).

Proof. Take all critical points of $P$ not belonging to $\mathrm{TH}(T)$, connect their $P$-images to infinity with pairwise disjoint simple curves ("rays") avoiding $\mathrm{TH}(P(T))$, and pull them back to their critical points to construct a finite collection of cuts of the plane. Let $W$ be a complementary component of this collections of cuts containing $T$. Clearly, $P: W \rightarrow P(W)$ is a branched covering map. By the Riemann-Hurwitz formula, the topological degree of this map is $m$ (observe that by the construction and by the assumptions and there are no critical points in $W \backslash T$ ). Thus points of $P(T)$ can have at most $m$ preimages in $T$. By the assumptions, this implies that they have exactly $m$ preimages in $T$ counting multiplicities.

Let us show that $T$ is a component of the set $P^{-1}(P(T))$. Indeed, by the previous paragraph condition (4) from Lemma 4.1 is fulfilled; hence, Lemma 4.1 implies the desired. Moreover, $T$ is the unique component of the set $P^{-1}(P(T))$ in $W$ (recall that the topological degree of $\left.P\right|_{W}$ is $m)$. Let us show that $P(\mathrm{TH}(T)) \subset \mathrm{TH}(P(T))$. Indeed, suppose otherwise. Then there exists a component $V$ of $\mathrm{TH}(T) \backslash T$ and a point $x \in V$ such that $P(x) \notin P(\mathrm{TH}(T))$. Connect $P(x)$ to infinity with a simple curve ("ray") avoiding $\mathrm{TH}(P(T))$ and pull it back to $x$. This gives rise to a "ray" connecting $x$ to infinity and avoiding $T$, a contradiction. Thus, $P(\mathrm{TH}(T)) \subset \mathrm{TH}(P(T))$.

Now, suppose that a point $y \in \mathrm{TH}(P(T))$ has a preimage $z \in W \backslash$ $\mathrm{TH}(T)$. By the above, this can only happen if $y \in \mathrm{TH}(P(T)) \backslash P(T)$. Let $U$ be the component of $\mathrm{TH}(P(T)) \backslash P(T)$ such that $y \in U$. Consider a component $Q$ of $P^{-1}(U)$ such that $z \in Q$. Since $z \notin \mathrm{TH}(T)$, it follows that there are points of $\operatorname{Bd}(Q) \subset W$ which do not belong to $T$ while their images belong to $\operatorname{Bd}(U) \subset T$. This contradicts the fact that $T$ is the unique component of the set $P^{-1}(P(T))$ in $W$ and completes the proof of the lemma).

Theorem B is the main result of this section. Recall that a parabolic domain at a periodic parabolic point $y$ is a periodic Fatou component 
that contains $y$ in its boundary and whose points converge to $y$ under the iterates of the given polynomial.

Theorem B. Let $P: \mathbb{C} \rightarrow \mathbb{C}$ be a polynomial, and $Y \subset \mathbb{C}$ be a full $P$-invariant continuum. The following assertions are equivalent:

(1) the set $Y$ is the filled Julia set of some polynomial-like map $P: U^{*} \rightarrow V^{*}$ of degree $k$,

(2) $Y$ is a component of the set $P^{-1}(P(Y))$, and, for every attracting or parabolic point $y$ of $P$ in $Y$, the immediate attracting basin of $y$ or the union of all parabolic domains at $y$ is a subset of $Y$.

The proof uses some ideas communicated by M. Lyubich to the fourth named author.

Proof. It suffices to prove $(2) \Longrightarrow(1)$. Let $\phi: \mathbb{D} \rightarrow \mathbb{C}^{*} \backslash Y$ be a Riemann map. By Lemma 4.1, a point $x \notin Y$ close to $Y$ cannot map into $Y$. Hence we can choose $\varepsilon>0$ so that the map $F=\phi^{-1} \circ P \circ \phi$ is defined and holomorphic on the annulus $A_{\varepsilon}=\{z: 1-\varepsilon<|z|<1\}$. Moreover, the map $F$ extends continuously to the unit circle $\{|z|=1\}$. Indeed, the map $\phi$ induces a homeomorphism $\widehat{\phi}$ between the set of prime ends of $\mathbb{C}^{*} \backslash Y$ and the unit circle. Note that $P$ induces a continuous map $\widehat{P}$ on the prime ends of $\mathbb{C}^{*} \backslash Y$. The continuous extension of $F$ is obtained by conjugating the map $\widehat{P}$ by the homeomorphism $\widehat{\phi}$.

By the Schwarz reflection principle, we can extend the map $F$ to a holomorphic map of the annulus $1-\varepsilon<|z|<(1-\varepsilon)^{-1}$ to $\mathbb{C}$ preserving $\mathbb{S}$ (hence taking this annulus to another annulus around $\mathbb{S}$ ). By a theorem of Mañé [Mañ85, if $F$ has no attracting or parabolic periodic points on $\mathbb{S}$, and no critical points on $\mathbb{S}$, then $F$ is expanding, i.e., $\left|\left(F^{\circ n}\right)^{\prime}(z)\right| \geqslant C \mu^{n}$ for some $C>0$ and $\mu>1$.

Since $F$ takes $A_{\varepsilon}$ to a subset of the disk $|z|<1$, it has no critical points on $\mathbb{S}$. Suppose that $F$ has an attracting or a parabolic periodic point $z$ of period $r$ on $\mathbb{S}$. In both cases, there is a convex Jordan domain $\widetilde{E}$ such that $F^{\circ r}(\widetilde{E}) \subset \widetilde{E}$, the closure of $\widetilde{E}$ contains $z$, and all points of $\widetilde{E}$ converge to $z$ under the iterations of $F^{\circ r}$. Since the unit circle is invariant under $F$, by the local theory of parabolic points, we can arrange that $\widetilde{E} \cap \mathbb{D} \neq \varnothing$. Note that $\widetilde{E}$ and the unit disk intersect over a convex Jordan domain $E$. By definition, $F^{\circ r}(E) \subset E$, and all points in $E$ converge to $z$ under the iterations of $F^{\circ r}$.

Set $B=\phi(E)$. Then $P^{\circ r}(B) \subset B$. By the Denjoy-Wolff theorem, all points of $B$ converge under the iterations of $P^{\circ r}$ to a $P^{\circ \text {-fixed point }}$ $x \in \operatorname{Bd}(B)$. Clearly, $x \in Y$, and $x$ is either attracting or parabolic (as it attracts an open set of points). However, by the assumptions, the 
attracting basin of $x$ or the union of all parabolic domains at $x$ is a subset of $Y$, a contradiction.

Thus, $F$ expands on $\mathbb{S}$, and $\varepsilon$ can be chosen so that the $F$-pullback of $A_{\varepsilon}$ is compactly contained in $A_{\varepsilon}$. Let $V^{*}$ be the Jordan domain bounded by the $\phi$-image of the curve $|z|=1-\varepsilon$. Set $U^{*}$ to be the component of $P^{-1}\left(V^{*}\right)$ containing $Y$. Then $\overline{U^{*}} \subset V^{*}$, and $P: U^{*} \rightarrow V^{*}$ is a polynomial-like map. The fact that $Y$ is the filled Julia set of this polynomial-like map, follows easily.

\section{Proof of Theorem C}

First we study the unbounded potentially renormalizable component.

Corollary 5.1. The unbounded potentially renormalizable component consists of immediately renormalizable maps.

Proof. Let $\mathcal{W}_{\infty}$ be the unbounded potentially renormalizable component in $\mathcal{F}_{\lambda}$. Then there is a polynomial $f \in \mathcal{W}_{\infty}$, whose Julia set is disconnected. Such a polynomial is necessarily immediately renormalizable, by $[\mathrm{BrHu}$, Theorem 5.3]. By Theorem A, it follows that for any $g \in \mathcal{W}_{\infty}$ the restriction $\left.g\right|_{X(g)}$ is quasi-symmetrically conjugate to $\left.f\right|_{X(f)}$ and therefore, in fact, to a quadratic polynomial from the Main Cardioid restricted on its Julia set. Thus, condition (1) of Theorem $\mathrm{B}$ is satisfied. Moreover, it follows that $\mathrm{TH}(X(g))$ does not contain attracting periodic points except for, possibly, 0. Finally, by Theorem A, it follows that $X(g)$ contains no parabolic periodic points except, possibly, for 0. Thus, condition (2) of Theorem B is satisfied too. Applying Theorem B to $g$, we see that $\left.g\right|_{X(g)}$ is quadratic-like and $g$ is immediately renormalizable as desired.

The following useful lemma was suggested to us by the referee which we gratefully acknowledge here.

Lemma 5.2. Let $\mathcal{V} \subset \mathcal{F}_{\lambda}$ be a stable component. Then no map from $\mathcal{V}$ can have a parabolic periodic point other than, possibly, 0 .

Proof. Suppose that $g \in \mathcal{V}$ has a parabolic periodic point $\zeta(g) \neq 0$. Then $g$ is isolated in the set of parameters in $\mathcal{F}_{\lambda}$ such that for the associated maps the point corresponding to $\zeta(g)$ is parabolic. Thus, in a small punctured neighborhood of $g$ in $\mathcal{F}_{\lambda}$ the multiplier of the associated map at the point corresponding to $\zeta(g)$, has absolute value greater than 1. By the Maximum Principle this implies that $\zeta(g)$ cannot be parabolic.

Let us now study bounded potentially renormalizable components. We need the notion of an active critical point introduced by McMullen 
in McM00]. Set $i=1$ or 2 , and take $f \in \mathcal{F}_{\lambda}$. The critical point $\omega_{i}(f)$ is active if, for every neighborhood $\mathcal{U}$ of $f$ in $\mathcal{F}_{\lambda}$, the sequence of the mappings $g \mapsto g^{\circ n}\left(\omega_{i}(g)\right)$ fails to be normal in $\mathcal{U}$. If the critical point $\omega_{i}(f)$ is not active, then it is said to be passive.

Corollary 5.3. Let $|\lambda| \leqslant 1$. Every bounded potentially renormalizable component $\mathcal{W}$ in $\mathcal{F}_{\lambda}$ consists of stable maps. If $\mathcal{W}$ contains an immediately renormalizable map, then it coincides with a stable component. The union $\mathcal{T}_{\lambda}$ of $\mathcal{P}_{\lambda}$ with all domains of stability, whose boundaries are contained in $\mathcal{P}_{\lambda}$, equals $\mathrm{TH}\left(\mathcal{P}_{\lambda}\right)$.

Proof. By [MSS83], to prove that $f \in \mathcal{W}$ is stable, it suffices to show that both critical points of $f$ are passive. Note that, if $g \in \operatorname{Bd}(\mathcal{W})$, then the $g$-orbits of $\omega_{1}(g)$ and of $\omega_{2}(g)$ are bounded uniformly with respect to $g$. By the maximum principle, the $f$-orbits of $\omega_{1}(f)$ and $\omega_{2}(f)$ are uniformly bounded for all $f \in \mathcal{W}$, which implies normality. Thus both critical points are passive, and the first claim of the corollary is proved.

Consider now a stable component $\mathcal{V}$ containing an immediately renormalizable polynomial $f$. We claim that then no polynomial $g \in \mathcal{P}_{\lambda} \cap \mathcal{V}$ exists. Indeed, suppose otherwise. The fact that $f$ is immediately renormalizable implies by Lemma 3.12 that $X(f)=J^{*}$ is its quadraticlike Julia set. Since $\mathcal{V}$ is stable, then $\left.g\right|_{J(g)}$ and $\left.f\right|_{J(f)}$ are conjugate. Let $Y$ be the continuum corresponding to $X(f)$ under this conjugacy. We want to apply to $Y$ Theorem B. Indeed, by construction of $Y$ and properties of $X(f)$ it follows that $g(Y)=Y$ and $Y$ is a component of the set $f^{-1}(Y)$.

Let us show that $g$ does not have any attracting periodic orbits other than possibly 0 . Indeed, suppose otherwise. Then, since any attracting periodic orbit persists under small perturbations, it follows that arbitrarily close to $g$ there are polynomials $h$ with $[h] \in \overline{\mathrm{PHD}_{3}}$ and an attracting periodic orbit distinct from $\{0\}$, a contradiction. Moreover, by Lemma $5.2 \mathrm{~g}$ cannot have a parabolic periodic point not equal to 0.

Thus, Theorem B applies to $Y$ and implies that $Y$ is a quadratic-like Julia set. Clearly, this contradicts the assumption that $g \in \mathcal{P}_{\lambda}$. Thus, if $\mathcal{V}$ is a stable component containing an immediately renormalizable polynomial then no polynomial $g \in \mathcal{P}_{\lambda} \cap \mathcal{V}$ exists. Since by Corollary 5.1 all polynomials in $\mathbb{C} \backslash \mathrm{TH}\left(\mathcal{P}_{\lambda}\right)$ are immediately renormalizable, it follows that stable components are either contained in $\mathcal{P}_{\lambda}$, or contained in $\mathbb{C} \backslash \mathcal{P}_{\lambda}$. In particular, the union $\mathcal{T}_{\lambda}$ of $\mathcal{P}_{\lambda}$ with all domains of stability, whose boundaries are contained in $\mathcal{P}_{\lambda}$, equals $\mathrm{TH}\left(\mathcal{P}_{\lambda}\right)$ (recall that by the above every bounded potentially renormalizable component $\mathcal{W}$ in $\mathcal{F}_{\lambda}$ consists of stable maps). 
We do not prove that all components of $\mathrm{TH}\left(\mathcal{P}_{\lambda}\right) \backslash \mathcal{P}_{\lambda}$ are stable components. This we can guarantee only for the components containing an immediately renormalizable map. Otherwise it might happen that a component $\mathcal{W}$ of $\operatorname{TH}\left(\mathcal{P}_{\lambda}\right) \backslash \mathcal{P}_{\lambda}$ is a proper subset of a stable component $\mathcal{V}$. However, we do prove that every stable component is contained in $\operatorname{TH}\left(\mathcal{P}_{\lambda}\right)$. Clearly, Corollary 5.3 together with Theorem 1.3 implies Lemma 1.4.

Corollary 5.4. Let $f \in \mathcal{U}$ where $\mathcal{U}$ is a bounded component of $\operatorname{TH}\left(\mathcal{P}_{\lambda}\right) \backslash$ $\mathcal{P}_{\lambda}$. Then $f$ does not have non-repelling periodic points except, possibly, for 0 . Moreover, $f$ does not have repelling periodic cutpoints in $J(f)$.

Proof. By Lemma [1.4, $[f] \in \mathrm{CU}$. Hence $f$ does not have repelling periodic cutpoints in $J(f)$. Suppose that $f$ has a non-repelling periodic point $y \neq 0$. Since $[f] \in \mathrm{CU}$, then the only possibility for $y$ is that $y$ is parabolic. However this contradicts the fact that $f$ belongs to a stable component (which follows from Corollary 5.3) and Lemma 5.2.

We are ready to discern in which bounded potentially renormalizable components maps are immediately renormalizable.

Definition 5.5. Assume that $f$ is immediately renormalizable with filled quadratic-like Julia set $K^{*}$. If there exists the smallest $n$ such that $f^{\circ n}\left(\omega_{2}(f)\right) \in K^{*}$, then we say that $f$ is an immediately renormalizable polynomial of capture type. Denote $n$ above by $n_{f}$; denote by $Q_{f}$ the pullback of $K^{*}$ containing $\omega_{2}(f)$.

Observe that $n_{f}>0$ by the definition of a quadratic-like map. Also, pullbacks of $K^{*}$ either coincide with $K^{*}$ or are disjoint from $K^{*}$. In particular, $Q_{f}$ is disjoint from $K^{*}$ by the definition of a pullback. All pullbacks of $K^{*}$ form a family of pairwise disjoint subcontinua of $K(f)$.

Lemma 5.6. Let $f$ be an immediately renormalizable polynomial of capture type. Then $n_{f}>1$.

Proof. If $n_{f}=1$, then $f\left(\omega_{2}(f)\right) \in K^{*}$ will have two preimages in $K^{*}$ and two more preimages in $Q_{f}$, a contradiction (recall that $f$ is cubic and that we count preimages with multiplicities).

Lemma 5.7 is used in the proof of Theorem C.

Lemma 5.7. An immediately renormalizable polynomial of capture type cannot belong to a bounded potentially renormalizable component.

Proof. Let $f \in \mathcal{F}_{\lambda},|\lambda| \leqslant 1$, belong to a bounded potentially renormalizable component $\mathcal{W}_{f}=\mathcal{W}$. Assume that $f$ is of capture type and 
is immediately renormalizable. The set $K^{*}$ and all its pullbacks contain lots of repelling periodic points and their preimages (such points are dense in the corresponding quadratic-like Julia set $J^{*}$ and its pullbacks). Obviously, we can choose two rays $R_{x}, R_{y}$ with arguments $\alpha$ and $\beta$ landing at preperiodic points $x, y \in Q_{f}$ such that $f(x)=f(y)$ and $f\left(R_{x}\right)=f\left(R_{y}\right)$. The union $R_{x} \cup Q_{f} \cup R_{y}$ cuts the plane into two pieces denoted by $L$ and $T$. Assume that $K^{*} \subset L$. Since $\left.f\right|_{K^{*}}$ is twoto-one, we may then assume that the $\operatorname{arc}(\alpha, \beta) \subset \mathbb{S}=\mathbb{R} / \mathbb{Z}$ is of length $\frac{2}{3}$ and contains all angles, whose rays are contained in $L$.

Set $F=f^{\circ\left(n_{f}-1\right)}$. Clearly, $Z=F\left(Q_{f}\right) \subset T$ (observe that $f(Z)=K^{*}$ while $Z$ and $K^{*}$ are disjoint) because, by construction, $f(T)$ covers $K^{*}$ while $f^{-1}\left(K^{*}\right)$ consists of two components, $K^{*}$ and $Z$. By Theorem 7.5.2 of [BFMOT12], this implies that $T$ contains either a repelling $F$-fixed cutpoint of $J(f)$ or a non-repelling $F$-fixed point. A particular case of [BFMOT12, Theorem 7.5.2] that is enough in our case states roughly the following: if a continuum $T$ is carved out of $K(f)$ by several "cut continua" mapping "towards" $T$ under $F$, then $T$ contains a repelling fixed cutpoint or a non-repelling fixed point for $F$. This contradicts Corollary 5.4 and completes the proof.

We are ready to prove Theorem $\mathrm{C}$.

Proof of Theorem C. Claim (1) of Theorem C is established in Corollary 5.1. Claim (2) of Theorem $\mathrm{C}$ follows from Lemma 5.7.

Consider now claim (3) of Theorem C. Let $f \in \mathcal{W}$ be a potentially renormalizable polynomial of queer type. By definition, this implies that $\omega_{2}(f) \in J(f)$. Clearly, if $\omega_{2}(f) \in X(f)$, then $f$ is not immediately renormalizable (by Lemma 3.12, if $f$ is immediately renormalizable, then its quadratic-like Julia set coincides with $X(f)$, and $\omega_{1}(f)$ is the unique critical point in $\mathrm{TH}(X(f)))$. Now, assume that $\omega_{2}(f) \notin X(f)$. Since $\mathrm{TH}(X(f)) \backslash X(f)$ is disjoint from $J(f)$, then $\omega_{2}(f) \notin \mathrm{TH}(X(f))$. Suppose that $f\left(\omega_{2}(f)\right) \in X(f)$. Then $f\left(\omega_{2}(f)\right)$ has at least four preimages (counting with multiplicities): at least two in $X(f)$ and $\omega_{2}(f)$ counted twice, a contradiction. Hence $f\left(\omega_{2}(f)\right) \notin X(f)$, and, since $\mathrm{TH}(X(f)) \backslash X(f)$ is disjoint from $J(f)$, then $f\left(\omega_{2}(f)\right) \notin \mathrm{TH}(X(f))$.

It follows now from Lemma 4.2 that the set $\mathrm{TH}(X(f))$ is a component of the set $f^{-1}(f(\mathrm{TH}(X(f)))$. Moreover, by Corollary 5.4, the polynomial $f$ does not have non-repelling periodic points except, possibly, for 0 . Hence Theorem B applies to $\mathrm{TH}(X(f))$ and shows that $f$ is immediately renormalizable. By Lemma 5.7, all this implies that the entire orbit of $\omega_{2}(f)$ is disjoint from $\mathrm{TH}(X(f))$ as desired.

On the other hand, by Lemma 5.7 a bounded potentially renormalizable component of Siegel capture type cannot contain an immediately 
renormalizable polynomial. Since by Theorem 1.6 a potentially renormalizable component is either of queer type or of Siegel capture type, this completes the proof of Theorem C.

\section{ACKNOWLEDGEMENTS}

We are grateful to M. Lyubich for useful discussions. We would also like to thank the referee for extensive suggestions, which have led to a significant improvement of the paper.

The first and the third named authors were partially supported by NSF grant DMS-1201450. The second named author was partially supported by NSF grant DMS-0906316. The fourth named author was partially supported by the Dynasty Foundation grant, RFBR grants 13-01-12449, 13-01-00969. The research comprised in Theorem B was funded by RScF grant 14-21-00053.

\section{REFERENCES}

[BFMOT12] A. Blokh, R. Fokkink, J. Mayer, L. Oversteegen, E. Tymchatyn, Fixed point theorems for plane continua with applications, Memoirs of the American Mathematical Society, 224 (2013), no. 1053

[BOPT14] A. Blokh, L. Oversteegen, R. Ptacek, V. Timorin, The main cubioid, Nonlinearity, 27 (2014), 1879-1897

[BOPT14a] A. Blokh, L. Oversteegen, R. Ptacek, V. Timorin, Complementary components to the cubic Principal Hyperbolic Domain, preprint, arXiv:1411.2535 (2014)

[BrHu] B. Branner, J. Hubbard, "The iteration of cubic polynomials, Part II: patterns and parapatterns", Acta Mathematica 169 (1992), 229-325.

[BuHe01] X. Buff, C. Henriksen, Julia Sets in Parameter Spaces, Commun. Math. Phys. 220 (2001), $333-375$

[DH8485] A. Douady and J. Hubbard, Étude dynamique des polynômes complex I \& II Publ. Math. Orsay (1984-85)

[DH85] A. Douady and J. Hubbard, On the dynamics of polynomial-like mappings, Ann. Sci. Éc. Norm. Sup. 18, 287-343 (1985)

[Hub93] J. H. Hubbard, "Local connectivity of Julia sets and bifurcation loci: three theorems of Yoccoz", in: Topological Methods in Modern Mathematics, Publish or Perish (1993)

[Lev91] G. Levin, On Pommerenkes inequality for the eigenvalues of fixed points, Colloq. Math. 62 (1991), 167-177.

[Lyu83] M. Lyubich, Some typical properties of the dynamics of rational mappings, Russian Math. Surveys 38 (1983), no. 5, 154-155.

[Mañ85] R. Mañé, Hyperbolicity, Sinks and Measure in One Dimensional Dynamics, Commun. Math. Phys. 100 (1985), 495-524.

[Mañ93] R. Mañé, On a theorem of Fatou, Bol. Soc. Bras. Mat. 24 (1993), 1-11.

[MSS83] R. Mañé, P. Sad, D. Sullivan, On the dynamics of rational maps, Ann. Sci. École Norm. Sup. (4) 16 (1983), no. 2, 193-217. 
[McM94] C. McMullen, Complex Dynamics and Renormalization, Princeton University Press, 1994.

[McM00] C. McMullen, The Mandelbrot set is universal, In: The Mandelbrot Set, Theme and Variations, Ed: Tan Lei. London Mathematical Society Lecture Note Series, 274, 1-18. Cambridge Univ. Press.

[Pet93] C. Petersen, On the Pommerenke-Levin-Yoccoz inequality, Ergod. Th. and Dynam. Sys. 13 (1993), 785-806.

[PeTa09] C. Petersen, Tan Lei, Analytic coordinates recording cubic dynamics, in: Complex Dynamics, Families and Friends, ed: D. Schleicher, A K Peters (2009).

[PiTa04] K. Pilgrim, Tan Lei, Spinning deformations of rational maps, Conformal Geometry and Dynamics 8 (2004), 52-86.

[Pom86] Ch. Pommerenke, On conformal mapping and iteration of rational functions, Complex Variables Theory Appl. 5 (1986), 117-126.

[Zak99] S. Zakeri, Dynamics of Cubic Siegel Polynomials, Commun. Math. Phys. 206 (1999), 185-233

(Alexander Blokh, Lex Oversteegen and Ross Ptacek) Department of Mathematics, University of Alabama at Birmingham, Birmingham, AL 35294

(Vladlen Timorin) Faculty of Mathematics, Laboratory of Algebraic Geometry and its Applications, Higher School of Economics, Vavilova St. 7, 112312 Moscow, Russia

(Vladlen Timorin) IndePendent University of Moscow, Bolshoy Vlasyevskiy Pereulok 11, 119002 Moscow, Russia

E-mail address, Alexander Blokh: ablokh@math.uab.edu

E-mail address, Lex Oversteegen: overstee@math.uab.edu

E-mail address, Ross Ptacek: rptacek@uab.edu

E-mail address, Vladlen Timorin: vtimorin@hse.ru 